\title{
Interstellar processes: Ortho/para conversion, radiative association, and dissociative recombination
}

\author{
Eric Herbst $t^{\mathrm{a}}$ \\ Departments of Chemistry and Astronomy, University of Virginia, Charlottesville, VA 22904, USA
}

\begin{abstract}
The study of the ortho-to-para ratio of assorted gas-phase interstellar molecules such as $\mathrm{H}_{2}, \mathrm{H}_{2} \mathrm{O}, \mathrm{NH}_{3}$, and $\mathrm{H}_{2} \mathrm{O}^{+}$has gained interest in recent years, based partially on new spectral observations of light hydrides by the Herschel Space Observatory. Although these ratios can yield valuable information about the thermal history of the interstellar cloud where the molecules are found, an understanding of how the ratios are determined involves a number of often poorly studied processes, which can include both gas-phase and grain-surface reactions. In this article, we consider the processes that determine the ortho-to-para ratio of the molecular ion $\mathrm{H}_{2} \mathrm{O}^{+}$in diffuse interstellar clouds and attempt to reproduce an unusual observed ratio for this ion. In addition to the study of ortho-topara ratios, we look carefully at current uncertainties in the gas-phase formation of large neutral molecules in cold dense interstellar clouds via ion-neutral radiative association and dissociative recombination, among other processes.
\end{abstract}

\section{Introduction}

The space among stars in our galaxy and others is not empty, but is populated by diverse objects consisting of gas and tiny dust particles known, for want of a better term, as interstellar clouds [1, 2]. The gas is dominated in one form or another by the element hydrogen, whereas the dust particles consist of either silicates or amorphous carbon, with mantles of ices in cold regions. These clouds derive from old stars, which undergo mass loss, either explosively, as in the case of supernovae, or more gently. The gas and dust that emerge from the atmospheres of these stars eventually condense under the influence of gravity to form rather transparent whispy objects known as diffuse clouds, which are relatively transparent to background starlight and have total gas densities of $10-100 \mathrm{~cm}^{-3}$, mainly in neutral atomic and molecular form. Eventually, some of these objects collapse to form so-called dense interstellar clouds, which are rather inhomogeneous and contain cold dense portions of material known as cold cores, dark cores, or pre-stellar cores. The cores have gas densities of $10^{3-4} \mathrm{~cm}^{-3}$ with a kinetic temperature of $10 \mathrm{~K}$, and are not transparent to visible and ultra-violet radiation. Most of the hydrogen is in molecular form, and significant numbers of complex but unusual gaseous molecules (cations, anions, radicals, isomers, and unsaturated hydrocarbons) through 13 atoms in size have been found mainly by the technique of rotational emission spectroscopy. In addition, simple ices such as water, $\mathrm{CO}, \mathrm{CO}_{2}$, and methanol have been detected via infrared absorption spectroscopy [3]. Although some cold cores

\footnotetext{
${ }^{a}$ Corresponding author: eh2ef@virginia.edu
}

This is an Open Access article distributed under the terms of the Creative Commons Attribution License 4.0, which permits unrestricted use, distribution, and reproduction in any medium, provided the original work is properly cited. 


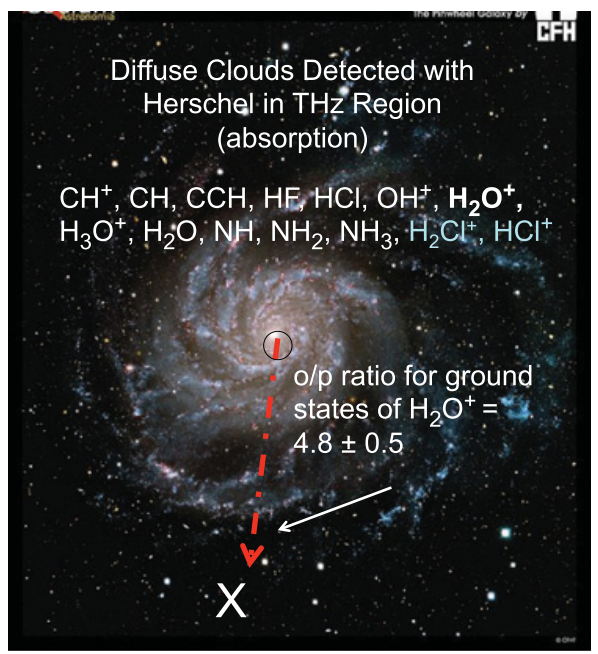

Figure 1. Absorption of far-infrared radiation from the center of our galaxy by diffuse clouds in the spiral arms. Note that the actual picture is of another spiral galaxy, the pinwheel galaxy, for purposes of illustration. Molecules detected by Herschel Space Observatory. Credit for CFHT Image: Canada-France-Hawaii Telescope/ J.-C. Cuillandre/Coelum.

appear to be stable, otherwise are in the process of collapse to form protostellar cores. The collapse starts isothermally, because sufficient radiation can escape to maintain a constant low temperature, but eventually the central condensation gets sufficiently dense to become optically thick. Further collapse then leads to adiabatic conditions, and the central temperature rises rapidly. This stage is often referred to as a protostar. Material continues to collapse inward towards the central object, which will eventually become a star. When the collapsing material reaches a temperature of $100-200 \mathrm{~K}$, it is often referred to as a hot core or corino, and the molecules detected in the gas become much more terrrestrial-like. At the same time, an accretion, or protoplanetary disk begins to form. Eventually, the stellar-disk system can evolve into a planetary system, as the dust particles condense into larger solid objects. The detailed evolutionary track discussed above more rigorously pertains to low-mass stars such as our Sun; the formation of high-mass stars is less well understood because of both faster evolution through assorted stages and the relative rarity of these objects.

Until recently, diffuse interstellar clouds were studied mainly by visible and ultra-violet absorption through relatively nearby clouds [4]. The gas was found to be mainly atomic with the exception of hydrogen, which is roughly evenly divided between atomic and molecular forms, while only a few other diatomic molecules were also detected. This picture has changed first with absorption studies using distant quasars as continuous millimeter-wave sources [5] and more recently with far-infrared (sometimes referred to as the $\mathrm{THz}$ region) absorption studies with the Herschel Space Observatory against continuua in this region of the spectrum, such as put out by hot cores [6]. Figure 1 shows a picture of radiation emitted from the center of an external galaxy, meant to represent our own, being absorbed by molecules in diffuse clouds in the spiral arms. Molecules detected with Herschel are listed, with new detections in light blue. The detections were part of a program known by the acronym PRISMAS, which stands for "Probing Interstellar Molecules with Absorption Line Studies." The water cation $\left(\mathrm{H}_{2} \mathrm{O}^{+}\right)$, detected in the spiral arms, was originally measured to have an ortho-to-para ratio of $4.8 \pm 0.5$, quite different from the normal value of 3.0 [6]. This unusual ratio has been of interest for some time, and we discussed it in a preliminary manner in a previous conference proceeding in this series [7]. In this paper, we consider the problem in more detail with the benefit of additional studies. 
The chemistry of $\mathrm{H}_{2} \mathrm{O}^{+}$in diffuse clouds is thought to be somewhat similar to that in cold cores, with initial ionization caused by cosmic ray (CR) bombardment of $\mathrm{H}$ and $\mathrm{H}_{2}$. Dissociative recombination plays a more important role, however, since the electron abundance is higher in diffuse clouds than in dense clouds due to the penetration of UV radiation. Some of the chemical reactions involved in the formation and depletion of the water cation and related species are as follows [8]:

$$
\begin{aligned}
& \mathrm{H}_{2}+\mathrm{CR} \longrightarrow \mathrm{H}_{2}^{+}+\mathrm{e}^{-}+\mathrm{CR} ; \mathrm{H}^{+}+\mathrm{H}+\mathrm{e}^{-}+\mathrm{CR}, \\
& \mathrm{H}+\mathrm{CR} \longrightarrow \mathrm{H}^{+}+\mathrm{e}^{-}+\mathrm{CR}, \\
& \mathrm{H}_{2}^{+}+\mathrm{H}_{2} \longrightarrow \mathrm{H}_{3}^{+}+\mathrm{H}, \\
& \mathrm{O}+\mathrm{H}^{+} \longrightarrow \mathrm{O}^{+}+\mathrm{H} \\
& \mathrm{O}^{+}+\mathrm{H}_{2} \longrightarrow \mathrm{OH}^{+}+\mathrm{H}, \\
& \mathrm{O}+\mathrm{H}_{3}^{+} \longrightarrow \mathrm{OH}^{+}+\mathrm{H}_{2} \\
& \mathrm{OH}^{+}+\mathrm{e}^{-} \longrightarrow \mathrm{O}+\mathrm{H} \\
& \mathrm{OH}^{+}+\mathrm{H}_{2} \longrightarrow \mathrm{o}, \mathrm{p}-\mathrm{H}_{2} \mathrm{O}^{+}+\mathrm{H} \text {, } \\
& \mathrm{H}_{2} \mathrm{O}^{+}+\mathrm{e} \longrightarrow \mathrm{OH}+\mathrm{H} ; \mathrm{O}+\mathrm{H}+\mathrm{H} ; \mathrm{O}+\mathrm{H}_{2}, \\
& \mathrm{H}_{2} \mathrm{O}^{+}+\mathrm{H}_{2} \longrightarrow \mathrm{H}_{3} \mathrm{O}^{+}+\mathrm{H} \text {, } \\
& \mathrm{H}_{3} \mathrm{O}^{+}+\mathrm{e}^{-} \longrightarrow \mathrm{OH}+2 \mathrm{H} ; \mathrm{H}_{2} \mathrm{O}+\mathrm{H} \text {, etc., }
\end{aligned}
$$

where the o and p in Eq. (8) refer to the ortho and para spin modifications of the water cation (see below). In order to produce a sufficient amount of water cation to reproduce the observation, it is necessary for the diffuse cloud to have much more atomic than molecular hydrogen, which is somewhat surprising since nearby diffuse clouds tend to have equal atomic and molecular abundances. An alternative interpretation is that the water cation is produced in the outer regions of the clouds, which are richer in atomic hydrogen than the inner regions [8].

\section{Ortho and para states}

For molecules with two indistinguishable protons (e.g. $\mathrm{H}_{2}, \mathrm{H}_{2} \mathrm{O}, \mathrm{H}_{2} \mathrm{O}^{+}$), the nuclear spin $I=1,0$ in the coupled representation, with the three symmetric $I=1$ states known as the ortho states, and the 


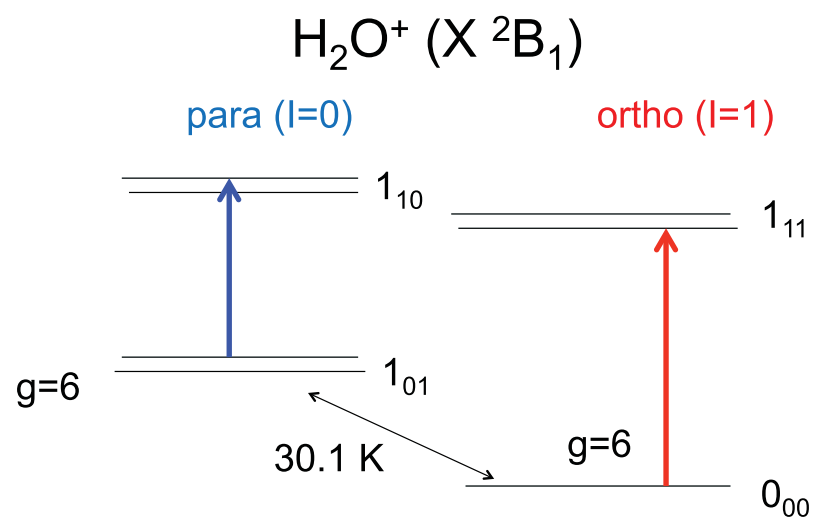

Figure 2. The lowest rotational states of the water cation in its ground electronic state divided into ortho and para groupings. Note that the fine and hyperfine structure lead to nearly degenerate groups of 6 states $(g=6)$ for the lowest rotational states. The arrows show some detected rotational transitions in diffuse interstellar clouds, where excited rotational states are likely not populated due to the low density of the gas. The $30.1 \mathrm{~K}$ refers to the energy difference between the lowest ortho and para states.

one antisymmetric $I=0$ state known as the para state [9]. According to the Pauli Exclusion Principle, the total wave function for two identical fermions must be antisymmetric to exchange. For the case of molecules, in the approximation that all degrees of freedom are separable, the overall wave function $\psi$ is given by the equation

$$
\psi=\psi_{\text {elec }} \psi_{\text {rot }} \psi_{n s},
$$

where "ns" stands for nuclear spin and the vibrational ground state can be neglected. This equation leads to two sets of rotational-nuclear spin states. For the common case in which the electronic wave function is symmetric to exchange, symmetric nuclear spin states (ortho) require antisymmetric rotational states, while antisymmetric nuclear spin states (para) require symmetric rotational states. The specific rotational states depend upon the structure of the molecules. For the diatomic molecule $\mathrm{H}_{2}$, the para states occur only with states of even rotational quantum number $J$, while the ortho states occur with states of odd rotational quantum number. For two-fold asymmetric tops with the symmetry axis along the $b$ principal axis and a symmetric electronic wave function, such as water, the para and ortho spin states occur with rotational states in which the two $K$ pseudo-quantum numbers, $K_{-1}$, and $K_{1}$, add to an even sum and an odd sum, respectively. For the water ion, however, the ground electronic wave function is of $X^{2} B_{1}$ symmetry, and is antisymmetric to proton exchange, so the ortho and para spin states occur with the opposite sums for the $K$ pseudo-quantum numbers. Figure 2 shows the lowest two ortho and para rotational states (with fine structure) for the water cation. The ortho rotational states also show hyperfine structure. The strongly allowed transitions maintain the nuclear spin (para-para, ortho-ortho); transitions between para and ortho rotational states are very weakly allowed, but possibly detectable if nuclear spin is coupled to electronic spin, as occurs in radicals or ions such as $\mathrm{H}_{2} \mathrm{O}^{+}$[10].

In the absence of any constraints, the number of ortho states populated should be three times the number of para states for molecules with two identical protons, so that the ortho-to-para ratio (OPR) is exactly three [11]. At temperatures significantly above the energy difference between the lowest ortho and para states, it is normally observed in the laboratory that the OPR is indeed three [9], [11]. If the ortho and para states are not coupled or coupled only very weakly by collisional and radiative mechanisms, this ratio will pertain as the temperature is lowered. If, on the other hand, there is strong 
coupling between the ortho and para forms of the molecule, which can occur in the gas by reactive collisions in which protons are exchanged: viz.

$$
\mathrm{H}_{\mathrm{a}} \mathrm{H}_{\mathrm{b}}+\mathrm{H}_{\mathrm{c}}^{+} \rightleftharpoons \mathrm{H}_{\mathrm{b}} \mathrm{H}_{\mathrm{c}}+\mathrm{H}_{\mathrm{a}}^{+},
$$

the OPR is governed by the equilibrium constant, $K_{\text {op }}$, between the two sets of states:

$$
\mathrm{OPR}=K_{\mathrm{op}}=\frac{q_{\text {ortho }}}{q_{\text {para }}} \exp (-\Delta E / T)
$$

where $\Delta E$ is the energy difference between the lowest ortho and para states, and the $q$ are partition functions over ortho and para states, with the zero of energy being the lowest ortho and para state, respectively. Note that for $\mathrm{H}_{2} \mathrm{O}^{+}$, the lowest ortho state is below the lowest para state, unlike the normal case, so that the energy difference is negative. At high temperatures compared with the energy difference, the equilibrium constant approaches three, while as the temperature approaches $0 \mathrm{~K}$, the partition functions reduce to the first term in the series, which is essentially the degeneracy of the lowest rotational state, neglecting fine and hyperfine structure. For $\mathrm{H}_{2} \mathrm{O}^{+}$, the ratio of partition functions then approaches unity, so that

$$
O P R=K_{\mathrm{op}} \approx \exp (30.1 / T) .
$$

Thus, if the system is at a temperature of $15 \mathrm{~K}$, the predicted equilibrium OPR is 7.4 , and it increases without limit as the temperature approaches $0 \mathrm{~K}$, unlike the normal equilibrium case, where it would approach zero.

\subsection{Interstellar complications}

One might expect that the environment in interstellar clouds would lead to an equilibrium OPR because ion-neutral reactions can indeed exchange protons, but there are two problems to be considered. First, the density must be compared with the so-called "critical" density, above which collisional effects dominate spontaneous emission so that inelastic collisions both excite and de-excite the rotational levels. In this case, rotation and translation approach the same temperature for polar species, as can occur in dense clouds [2]. In diffuse clouds, on the other hand, the most likely situation is that the rotational temperature is quite low for polar species, and can reach $2.7 \mathrm{~K}$, the black body temperature of the universe. In that case, we should only consider the lowest ortho and para rotational states so that the OPR for $\mathrm{H}_{2} \mathrm{O}^{+}$is given by Eq. (15) at all kinetic temperatures. Because the range of kinetic temperatures in diffuse clouds is $50-100 \mathrm{~K}$, the predicted equilibrium OPR is $1.4-1.8$, nowhere near the observed value of 4.8 . Another way of looking at the problem, however, is to use Eq. (15) to define a so-called spin temperature. To recover the observed OPR of 4.8 , the spin temperature would have to be $20 \mathrm{~K}$, which corresponds to no other temperature in a diffuse cloud, and is probably meaningless.

The second complication is that chemical equilibrium may not pertain, given the slow collision rates in diffuse clouds, so that the OPR is not given by an equilibrium constant, but is kinetically controlled. In this case, the rates and products of various processes control the value of the OPR for $\mathrm{H}_{2} \mathrm{O}^{+}$. The formation of $\mathrm{H}_{2} \mathrm{O}^{+}$occurs via the reaction of the precursor ion $\mathrm{OH}^{+}$and $\mathrm{H}_{2}$, which is shown in Eq. (8). This reaction possesses a near Langevin rate coefficient $\left(\approx 10^{-9} \mathrm{~cm}^{3} \mathrm{~s}^{-1}\right)$, given by the simple expression

$$
k=2 \pi e \sqrt{\alpha / \mu},
$$


Table 1. Fractions $f$ of ortho- and para- $\mathrm{H}_{2} \mathrm{O}^{+}$produced from $\mathrm{OH}^{+}$and o- and $\mathrm{p}-\mathrm{H}_{2}$.

\begin{tabular}{llccc}
\hline $\mathrm{H}_{2}$ & Mechanism & $\mathrm{o}-\mathrm{H}_{2} \mathrm{O}^{+}$ & $\mathrm{p}-\mathrm{H}_{2} \mathrm{O}^{+}$ & $\mathrm{OPR}$ \\
\hline ortho & scrambling & $5 / 6$ & $1 / 6$ & 5 \\
para & scrambling & $1 / 2$ & $1 / 2$ & 1 \\
ortho & $\mathrm{H}$ hopping & $3 / 4$ & $1 / 4$ & 3 \\
para & $\mathrm{H}$ hopping & $3 / 4$ & $1 / 4$ & 3 \\
\hline
\end{tabular}

where $e$ is the electronic charge in esu, $\alpha$ is the dipole polarizability of the neutral reactant in $\mathrm{cm}^{3}$, and $\mu$ is the reduced mass of the reactants in gm [12]. The fraction of ortho and para $\mathrm{H}_{2} \mathrm{O}^{+}$produced is determined both by the spin selection rules and by the fractions of the ortho and para $\mathrm{H}_{2}$ reactant. The spin selection rules have been studed theoretically by both Oka [13] and Quack [14]. Here we have used the somewhat simpler treatment of Oka, which is based on angular momentum considerations only, and is most applicable to very exothermic reactions in which a number of rotational states of the products can be produced. To complicate matters, the results depend upon the mechanism of reaction, and we have considered two different mechanisms. The first one, known as scrambling, pertains to the case of a long-lived intermediate complex, in which all routes that lead to products are allowed. The second is the case of $\mathrm{H}$ hopping, in which a hydrogen atom from $\mathrm{H}_{2}$ breaks off and attaches to the hydroxyl ion, presumably at long range. The fractions of o- and $\mathrm{p}-\mathrm{H}_{2} \mathrm{O}^{+}$produced in each situation are shown in Table 1. Following the reaction, the para and ortho forms of the water cation relax to form the lowest rotational states for each nuclear spin state (see Fig. 2) preserving the original OPR. The relaxation occurs mainly by spontaneous emission. Afterwards, a competition ensues between equilibration, which likely occurs most efficiently via collisions with abundant $\mathrm{H}$ atoms:

$$
\mathrm{O}-\mathrm{H}_{2} \mathrm{O}^{+}+\mathrm{H} \leftrightharpoons \mathrm{p}-\mathrm{H}_{2} \mathrm{O}^{+}+\mathrm{H}
$$

and destruction, which can occur via dissociative recombination (Eq. (9)) or by reaction with $\mathrm{H}_{2}$ (Eq. (10)). The estimated rate coefficients for these two modes of destruction at $50 \mathrm{~K}$ are $1.2 \times$ $10^{-6} \mathrm{~cm}^{3} \mathrm{~s}^{-1}$ for dissociative recombination and $6.4 \times 10^{-10} \mathrm{~cm}^{3} \mathrm{~s}^{-1}$ for reaction with $\mathrm{H}_{2}$ [8]. In diffuse clouds, the electron fractional abundance with respect to density is $\approx 10^{-4}$ while to reproduce the observed abundance of $\mathrm{H}_{2} \mathrm{O}^{+}$, the fractional abundance of $\mathrm{H}_{2}$ is $\approx 0.05$. Thus, dissociative recombination is likely the dominant destruction mechanism. The rate coefficient for collisions in which $\mathrm{H}$ atoms equilibrate o- and $\mathrm{p}-\mathrm{H}_{2} \mathrm{O}^{+}$is uncertain. Note that full equilibration would involve both translational energy and spin state. Translational energy equilibration can involve non-reactive as well as reactive collisions, whereas spin equilibration involves reactive collisions in which protons are exchanged. If we assume that these reactive collisions occur with a rate coefficient near the Langevin value, the amount of atomic hydrogen is large enough so that equilibration is somewhat faster than reaction with electrons, but if the spin conversion occurs at a rate that is slower than given by the Langevin value, it is unclear which process dominates, if either.

We have already seen that for the case in which equilibration dominates, the OPR for $\mathrm{H}_{2} \mathrm{O}^{+}$is predicted to lie between 1.4 and 1.8 in the kinetic temperature range 50-100 K. If, on the other hand, reactive destruction dominates, the formation reaction of $\mathrm{H}_{2} \mathrm{O}^{+}$has an overall rate coefficient that is independent of the spin state of $\mathrm{H}_{2}$, and the dissociative recombination process (Eq. (9)) has a rate coefficient that is independent of the spin state of $\mathrm{H}_{2} \mathrm{O}^{+}$, we can obtain the following formula for the OPR of the water cation at steady state [7]:

$$
O P R=\frac{f_{p o}+f_{o o} O P R\left(H_{2}\right)}{f_{p p}+f_{o p} O P R\left(H_{2}\right)}
$$


Table 2. Complex Organic Molecules Associated with Hot Cores and Corinos.

\begin{tabular}{lllll}
\hline O-Containing & O-Containing & N-Containing & N-Containing & N,O-Containing \\
\hline $\mathrm{CH}_{3} \mathrm{OH}$ & $\mathrm{HC}_{2} \mathrm{CHO}$ & $\mathrm{CH}_{3} \mathrm{CN}$ & $\mathrm{CH}_{3} \mathrm{NC}$ & $\mathrm{NH}_{2} \mathrm{CHO}$ \\
$\mathrm{c}-\mathrm{C}_{3} \mathrm{H}_{2} \mathrm{O}$ & $\mathrm{CH}_{3} \mathrm{CHO}$ & e- $\mathrm{HNCHCN}$ & $\mathrm{CH}_{2} \mathrm{CNH}$ & $\mathrm{CH}_{3} \mathrm{CONH}$ \\
$\mathrm{C}_{2} \mathrm{H}_{3} \mathrm{OH}$ & $\mathrm{c}-\mathrm{CH}_{2} \mathrm{OCH}$ & $\mathrm{CH}_{3} \mathrm{NH}_{2}$ & $\mathrm{C}_{2} \mathrm{H}_{3} \mathrm{CN}$ & $\mathrm{NH}_{2} \mathrm{CH}_{2} \mathrm{COOH}(? ?)$ \\
$\mathrm{HC}(\mathrm{O}) \mathrm{OCH}_{3}$ & $\mathrm{CH}_{3} \mathrm{COOH}$ & $\mathrm{NH}_{2} \mathrm{CH}_{2} \mathrm{CN}$ & $\mathrm{H}_{3} \mathrm{CCHNH}$ & \\
$\mathrm{HOCH} \mathrm{H}_{2} \mathrm{CHO}$ & $\mathrm{C}_{2} \mathrm{H}_{3} \mathrm{CHO}$ & $\mathrm{C}_{2} \mathrm{H}_{5} \mathrm{CN}$ & $\mathrm{C}_{3} \mathrm{H}_{7} \mathrm{CN}$ & \\
$\mathrm{C}_{2} \mathrm{H}_{5} \mathrm{OH}$ & $\mathrm{CH}_{3} \mathrm{OCH}_{3}$ & & & \\
$\mathrm{CH}_{3} \mathrm{COCH}$ & $\mathrm{HOCH}_{2} \mathrm{CH}_{2} \mathrm{OH}$ & & & \\
$\mathrm{C}_{2} \mathrm{H}_{5} \mathrm{CHO}$ & $\mathrm{HCOOC}_{2} \mathrm{H}_{5}$ & & & \\
$\mathrm{CH}_{3} \mathrm{C}(\mathrm{O}) \mathrm{OCH}_{3}$ & & & & \\
\hline
\end{tabular}

Here, $f_{x y}$ stands for the fraction of the $x$ spin modification of $\mathrm{H}_{2}$ that is converted into the $y$ spin modification of $\mathrm{H}_{2} \mathrm{O}^{+}$via Eq. (8), where $o$ and $p$ refer to ortho and para. The values obtained for $f$ are listed in Table 1 for both the scrambling and hopping mechanisms. The final parameter needed to solve Eq. (18) is the OPR for molecular hydrogen, which has been measured for a number of diffuse clouds to be in the range $0.2-0.5$. The resulting range of calculated OPR values for $\mathrm{H}_{2} \mathrm{O}^{+}$is $1.3-1.6$ for the scrambling mechanism and a fixed value of 3 for the $\mathrm{H}$ hopping mechanism. None of these values equals the observed value of 4.8 !

In a final attempt to reproduce the observed OPR in diffuse clouds toward the galactic center, Tanaka et al. [10] studied the possibility that, as the ortho and para forms of $\mathrm{H}_{2} \mathrm{O}^{+}$radiatively relax down their respective ladders following their formation in the exothermic reaction between $\mathrm{OH}^{+}$and $\mathrm{H}_{2}$, radiative transitions can occur between ortho and para states, especially for high rotational levels that are close to degeneracy. The effect is enhanced by an intense infrared radiation field that can maintain rotational excitation. In this possible but unlikely scenario, the $\mathrm{OPR}$ for $\mathrm{H}_{2} \mathrm{O}^{+}$can indeed reach 4.8. The unlikeliness of the scenario is mainly caused by the small number of cases in which diffuse clouds are in the neighborhood of sources of intense infrared radiation.

In an ironic twist, a new and improved observational study of $\mathrm{H}_{2} \mathrm{O}^{+}$in diffuse clouds towards the galactic center has resulted in a new value for the OPR of $3.2 \pm 0.4$ [15], which is consistent with a value of 3 . The observed value is, perhaps serendipitously, in agreement with the value we obtain for the case in which the destruction of the water cation by dissociative recombination is more rapid than thermalization of the spin, and the formation occurs via reaction (8) by the mechanism of $\mathrm{H}$ hopping rather than by scrambling. The problem is made more complex by the existence of other possible formation mechanisms for $\mathrm{H}_{2} \mathrm{O}^{+}$, such as the formation of water in ice mantles of interstellar grains followed by desorption into the gas and subsequent photoionization.

\section{Formation of complex organic molecules}

Although many of the detected gaseous interstellar molecules are exotic by terrestrial standards, others are well-known in the terrestrial laboratory, and include simple alcohols, organic acids, esters, ethers, and amines. These well-known species are generally known as "complex organic molecules", or COMs for short, if they have six or more atoms [3]. The species tend to be more saturated (hydrogen-rich) and most often contain oxygen or nitrogen as well as carbon and hydrogen. Table 2 contains a partial list of these species, which are mainly associated with hot cores while cold cores are mainly associated with the more exotic species such as radicals and unsaturated linear hydrocarbons, known in astronomy as carbon chains. Although the gas-phase and ice chemistries that occur in cold cores are relatively well understood, especially the gas-phase ion-neutral chemistry [16], the processes that form the COMs detected mainly in hot cores are still a subject of contention, and the simulations of the chemistry semiquantitative at best [3]. Most treatments involve production of COMs on granular ice mantles followed, as star-formation occurs, by sublimation into the gas. The surface chemistry can occur during the cold 
core stage, prior to star formation, or during the warm-up period as the pre-stellar core turns into a protostellar core. If the chemistry occurs on $10 \mathrm{~K}$ ice mantles, it is dominated by reactions involving atoms, because these are the most weakly bound species and are able to diffuse from binding site to binding site so as to find reactant partners. An example is the formation of methanol by the successive hydrogenation of $\mathrm{CO}$, formed initially in the gas, by $\mathrm{H}$ atoms on grain surfaces [17]:

$$
\mathrm{CO}+\mathrm{H} \rightarrow \mathrm{HCO} ; \mathrm{HCO}+\mathrm{H} \rightarrow \mathrm{H}_{2} \mathrm{CO} ; \mathrm{H}_{2} \mathrm{CO}+\mathrm{H} \rightarrow \mathrm{H}_{2} \mathrm{COH}\left(\mathrm{CH}_{3} \mathrm{O}\right) ; \mathrm{CH}_{3} \mathrm{O}+\mathrm{H} \rightarrow \mathrm{CH}_{3} \mathrm{OH} \text {. }
$$

However, little laboratory confirmation has been obtained for reaction pathways leading to grain species larger than methanol. There is more laboratory evidence for a pathway involving association reactions between radicals at higher temperatures [18], and a number of models have been reported with this mechanism [19].

As the temperature increases to $\approx 20-30 \mathrm{~K}$, molecules are also able to diffuse, although most of the species are unreactive unless converted into reactive radicals by photodissociation or particle bombardment on the grains. The radicals can recombine on grains to produce larger organic molecules. At grain temperatures significantly under $50 \mathrm{~K}$, only volatile species such as $\mathrm{CO}$ and methane can sublime; other species can desorb via non-thermal means such as photodesorption and reactive desorption, in which the exothermicity of reactions is utilized to break the adsorbate-surface bond. Once species are returned to the gas, they can undergo a new round of gas-phase chemistry. For example, ionneutral processes starting with methane can lead to the production of carbon chain species, in a similar manner to what happens at $10 \mathrm{~K}$ in cold cores. At temperatures exceeding $100 \mathrm{~K}$, on the other hand, near total sublimation of the molecules in the ice mantles occurs, and large abundances of COMs are detected in the gas. The warm-up process occurs during the collapse of material onto the protostellar core, so the details of the physical collapse affect the chemistry. Current chemical models actually treat the collapse with hydrodynamics; one-dimensional treatments [20] can be studied for longer periods of time than more recent three-dimensional treatments, which do show, however, the formation of a proto-disk [21].

Although COMs are observed at their highest abundances in hot cores and corinos, a few, such as methanol, methyl formate, dimethyl ether, and acetaldehyde have been observed at much lower abundances in a variety of other types of sources. These include portions of the galactic center, socalled infra-red dark clouds, which are clouds so massive that even infrared radiation cannot penetrate through them, active galactic nuclear disks, which surround massive black holes at the center of energetic galaxies, and, lastly, cold cores [22]. For some of these sources, warm-up models, similar to those used for hot cores, seem to simulate the chemistry reasonably well, with the lower abundances caused by non-thermal desorption from grains at lower temperatures than those pertaining to hot cores. But, the case of cold cores is unique, because warm-up models are not relevant to a cloud at $10 \mathrm{~K}$, where grain surfaces are too cold for molecules to undergo diffusive reactions on granular surfaces. In the year or so since a number of COMs were detected in several cold cores, a variety of theoretical explanations have been proffered. These include

- High temperature fluctuations or non-thermal chemistry on dust particles.

- Synthesis on cold surfaces, one atom at a time.

- More efficient non-thermal desorption from grains followed by additional gas-phase reactions such as radiative association processes.

Of these three possibilities, the first could occur if, for example, a heavy cosmic ray collided with a grain, and raised the temperature, leading to molecular diffusion and possible reactions. The second has already been discussed, and does not have much support from laboratory studies done to date. Chemical simulations using the third approach have already appeared in the astrochemical literature [23, 24]. Here, the first necessity is to increase the rate of non-thermal desorption into the gas, which can be done assuming that the energy of exothermic chemical reactions on the surface can be channeled into breaking the molecular product-grain surface bond. This process, which has received support from a 
recent experiment [25], enriches the cold gas in precursor material for chemical reactions that can lead to COMs. For example, if formaldehyde is produced on granular surfaces and ejected into the gas, it can be protonated by ion-neutral processes such as

$$
\mathrm{H}_{2} \mathrm{CO}+\mathrm{H}_{3}^{+} \longrightarrow \mathrm{H}_{2} \mathrm{COH}^{+}+\mathrm{H}_{2},
$$

after which the protonated formaldehyde cation can undergo a radiative association with formaldehyde to produce a large complex ion:

$$
\mathrm{H}_{2} \mathrm{COH}^{+}+\mathrm{H}_{2} \mathrm{CO} \longrightarrow \mathrm{C}_{2} \mathrm{H}_{5} \mathrm{O}_{2}^{+}+\mathrm{h} v \text {. }
$$

Unfortunately, radiative association processes have rarely been studied in the laboratory [26], and simple statistical theories are used to determine their rate coefficients, which are found to increase in size with increasing size of the product and increasing reaction exothermicity [27]. If the product ion in Eq. (21) has the structure of protonated methyl formate, then subsequent dissociative recombination should yield a significant two-body product channel that includes methyl formate:

$$
\mathrm{C}_{2} \mathrm{H}_{5} \mathrm{O}_{2}^{+}+\mathrm{e}^{-} \longrightarrow \mathrm{HC}(\mathrm{O}) \mathrm{OCH}_{3}+\mathrm{H} \text {. }
$$

But quantum chemical calculations show that the only product ion formed in reaction (21) without a transition state barrier has the structure $\left[\mathrm{H}_{2} \mathrm{COCH}_{2} \mathrm{OH}\right]^{+}$, which is not protonated methyl formate [28]. Indeed, the conversion of this ion to protonated methyl formate, $\left[\mathrm{HC}(\mathrm{OH}) \mathrm{OCH}_{3}\right]^{+}$, is predicted to have a substantial barrier. Whether or not the COM methyl formate can be formed by dissociative recombination of the ion $\left[\mathrm{H}_{2} \mathrm{COCH}_{2} \mathrm{OH}\right]^{+}$depends upon whether significant structural transformation can occur during the recombination to form a precursor neutral, which then dissociates. One structural transformation known to occur in dissociative recombination reactions involves the motion of hydrogen atoms; viz.,

$$
\mathrm{H}_{2} \mathrm{O}^{+}+\mathrm{e}^{-} \longrightarrow \mathrm{O}+\mathrm{H}_{2}
$$

where the two $\mathrm{H}$ atoms must break their bonds to the central $\mathrm{O}$ atom and form a new bond together [29]. More study, both experimental and theoretical, is needed to resolve this question for unstudied large molecular ions. If structural rearrangements can occur, statistical theories can be used to determine isomeric branching fractions, and then tested against branching ratios obtained in storage ring experiments. For example, in reaction (22), it is thermodynamically possible that neutral products can include the three different isomers methyl formate, acetic acid, and glycolaldhyde.

If it is assumed that reactive desorption can occur for $1-10 \%$ of the products of reactions on grains, and that radiative association and subsequent dissociative recombination processes can lead to products with diverse isomeric structures, then COMs such as methyl formate and dimethyl ether can be produced at abundances near to those observed in cold cores [23]. Predictions for the abundances of COMs other than the few detected so far in cold cores would be useful in checking and confirming this mechanism. A variant of the above idea has been suggested by Rawlings et al. [24] who investigated reactions that occur at transient higher densities as the desorption is actually occurring. This approach gains plausibility if the desorption mechanism is an explosion caused by a runaway chain-type reaction of radicals.

\section{Radiative recombination?}

As a neutral molecule or molecular ion gets larger, it becomes more difficult for it to be destroyed by photons, because the energy gained by the absorption of a photon gets spread out into numerous vibrational modes rather than all appearing in one mode that leads directly to dissociation. Instead, 
photons tend to ionize large molecules either directly in a single-photon event when the photon has sufficient energy to ionize a molecule, or indirectly via the sequential absorption of a number of less energetic photons. This second process results in a delayed ionization, and is analogous to the process of thermionic emission from bulk materials. A molecule for which both indirect photoionization and photofragmentation have been studied is buckminsterfullerene, or $\mathrm{C}_{60}$ [30]. Consider thermionic emission from a single molecule of $\mathrm{C}_{60}$, the ionization energy of which is $7.58 \mathrm{eV}$. If photoionization is caused by multiple photons, each with less energy than the ionization potential, the production of ions and electrons is delayed into the microsecond range. The process has been treated by a statistical model due to Klots [31]. Photo-fragmentation can also occur, either by a single photon or by multiple photons. The process is slow, however, and occurs mainly via $\mathrm{C}_{2}$ release.

What do these facts tell us about the electron recombination of $\mathrm{C}_{60}^{+}$? Imagine an electron colliding and sticking to a $\mathrm{C}_{60}^{+}$ion to form a long-live neutral complex $\mathrm{C}_{60}^{*}$. This complex can autoionize back to $\mathrm{C}_{60}^{+}$, can dissociate into fragments, leading to dissociative recombination, or can stabilize itself by radiation of infrared photons, leading to radiative recombination. At high densities of a background gas (irrelevant to the situation in interstellar clouds), ternary recombination can also occur, where the complex loses enough energy in collisions to stabilize itself. Since, for $\mathrm{C}_{60}$, ionization by multiple photons dominates over dissociation, microscopic reversibility apparently tells us that the process of radiative recombination via multiple photon emission is likely to dominate over dissociative recombination to form $\mathrm{C}_{58}$ and $\mathrm{C}_{2}$ :

$$
\mathrm{C}_{60}^{+}+\mathrm{e}^{-} \longrightarrow \mathrm{C}_{60}+\mathrm{h} v ; \mathrm{C}_{58}+\mathrm{C}_{2},
$$

where the 58-carbon species is still a fullerene. The statistical model of Klots can also be used to determine the branching fraction between radiative recombination and dissociative recombination, and we intend to pursue this calculation for $\mathrm{C}_{60}^{+}$and for other polyatomic ions of interstellar importance.

I wish to acknowledge support from the NASA-JPL program for the analysis of Herschel data, as well as support for my research program in astrochemistry by the National Science Foundation and by the NASA Exobiology and Evolutionary Biology program through a subcontract from Rensselaer Polytechnic Institute.

\section{References}

[1] B. T. Draine, Physics of the Interstellar and Intergalactic Medium (Princeton University Press, Princeton, NJ 2011)

[2] A. G. G. M. Tielens, The Physics and Chemistry of the Interstellar Medium (Cambridge University Press, Cambridge, UK 2005)

[3] E. Herbst and E. F. van Dishoeck, Annual Reviews of Astronomy \& Astrophysics 47 (2009) 427-480

[4] B. J. McCall, in Astrochemistry: Recent Successes and Current Challenges. IAU Symposium 231, ed. D. C. Lis, G. A. Blake, \& E. Herbst (Cambridge University Press, Cambridge, UK 2005) $155-174$

[5] H. S. Liszt, R. Lucas, \& J. Pety in Astrochemistry: Recent Successes and Current Challenges. IAU Symposium 231, ed. D. C. Lis, G. A. Blake, \& E. Herbst (Cambridge University Press, Cambridge, UK 2005) 187-196

[6] P. Schilke \& 49 co-authors, Astronomy \& Astrophysics 521 (2010) id. L11, 1-7

[7] E. Herbst and E. Roueff, J. Phys.: Conf. Ser. 300 (2011) 012026, 1-8

[8] N. Indriolo, D. A. Neufeld, M. Gerin, T. R. Geballe, J. H. Black, K. M. Menten, \& J. R. Goicoechea, Astrophys. J. 758 (2012) id. 83, 1-8

[9] W. Gordy and R. L. Cook, Techniques of Chemistry, Microwave Molecular Spectra (Vol. 18) (Wiley-Interscience, New York 1984) 
[10] K. Tanaka, K. Harada, and T. Oka, J. Phys. Chem. 117A (2013) 9584-9592

[11] C. H. Townes and A. L. Schawlow, Microwave Spectroscopy (Dover Publications, New York 1975)

[12] V. G. Anicich, J. Phys. Chem. Ref. Data 22 (1993) 1469-1569

[13] T. Oka, J. Molecular Spectroscopy 228 (2004) 635-639

[14] M. Quack, Molecular Physics 34 (1977) 477-504

[15] P. Schilke, D. C. Lis, E. A. Bergin, R. Higgins, and C. Comito, J. Phys. Chem. 117 (2013) 97669769

[16] R. T. Garrod, V. Wakelam, and E. Herbst, Astronomy \& Astrophysics 467 (2007) 1103-1115

[17] S. B. Charnley, A. G. G. M. Tielens, and S. D. Rodgers, Astrophys. J. 482 (1997) L203-L206

[18] K. I. Öberg, R. T. Garrod, E. F. van Dishoeck, and H. Linnartz, Astronomy \& Astrophysics 504 (2009) 891-913

[19] R. T. Garrod, S. L. Widicus Weaver, and E. Herbst, Astrophys. J. 682 (2008) 283-302

[20] Y. Aikawa, V. Wakelam, F. Hersant, R. T. Garrod, and E. Herbst, Astrophys. J. 760 (2012) id. 40, $1-19$

[21] U. Hincelin, V. Wakelam, B. Commercon, F. Hersant, and S Guilloteau, Astrophys. J., 775 (2013) id. $55,1-11$

[22] A. Bacmann, V. Tacquet, A. Faure, C. Kahane, and C. Ceccarelli, Astronomy \& Astrophysics 541 (2012) id. A12, 1-5

[23] A. I. Vasyunin and E. Herbst, Astrophys. J. 769 (2013) id. 34, 1-9

[24] J. M. C. Rawlings, D. A. Williams, S. Viti, C. Cecchi-Pestellini, and W. W. Duley, Monthly Not. R. Astr. Soc. 430 (2013) 264-273

[25] F. Dulieu, E. Congiu, J. Noble, S. Baouche, H. Chaabouni, A. Moudens, M. Minissale, and S. Cazaux, Nature Scientific Reports 3 (2013) i.d. 1338, 1-6

[26] D. Gerlich and S. Horning, Chem. Reviews 92 (1992) 1509-1539

[27] D. R. Bates and E. Herbst, in Rate Coefficients in Astrochemistry (Kluwer, Dordrecht 1988) 17-40

[28] A. Horn, H. Mollendal, O. Sekiguchi, E. Uggerud, H. Roberts, E. Herbst, A. A. Viggiano, and T. D. Fridgen, Astrophys. J. 611 (2004) 605-614

[29] S. Rosen, A. Derkatch, J. Semaniak, A. Neau, A. Al-Khalili, A. Le Padellec, L. Vikor, R. Thomas, H. Danared, M. af Ugglasd, and M. Larsson, Faraday Discussions 115 (2000) 295-302

[30] M. S. Dresselhaus, G. Dresselhaus, and P. C. Eklund, Science of Fullerenes and Carbon Nanotubes (Academic Press, San Diego 1996)

[31] C. E. Klots, Chem. Phys. Lett. 186 (1991) 73-76 